\title{
Role of Ultrasound in the Evaluation of Acute Pelvic Pain in Nonpregnant Reproductive Age Patients
}

\author{
Nadah Zafar, Sanja Kupesic Plavsic
}

\begin{abstract}
In an emergency setting pelvic ultrasound is the tool of choice for the evaluation of the patients presenting with acute pelvic pain. It has a high sensitivity and specificity for detection of pelvic pathology, low cost, widespread availability and lack of ionizing radiation. This article presents a variety of case scenarios regarding the nonpregnant female of reproductive age presenting with acute pelvic and lower abdominal pain. By using illustrative images, the reader will be able to differentiate between the most common causes of abdominal and/or pelvic pain and identify typical B-mode and color Doppler ultrasound findings and $\mathrm{CT}$ images that require immediate treatment, while minimizing the performance of unnecessary additional imaging and invasive procedures.
\end{abstract}

Keywords: Abdominal pain, Pelvic pain, Nonpregnant patients, Pelvic inflammatory disease, Adnexal torsion, Ovarian cyst, Appendicitis, Urolithiasis, Cystitis, Cholelithiasis, B-mode ultrasound, Color Doppler ultrasound.

How to cite this article: Zafar N, Kupesic Plavsic S. Role of Ultrasound in the Evaluation of Acute Pelvic Pain in Nonpregnant Reproductive Age Patients. Donald School J Ultrasound Obstet Gynecol 2012;6(2):207-217.

\section{Source of support: Nil}

Conflict of interest: None declared

\section{INTRODUCTION}

Acute pelvic and/or lower abdominal pain in nonpregnant women of reproductive age is an extremely common presentation to emergency departments, urgent care centers and outpatient office practices. Approximately 1.4 million gynecologic visits were made to emergency departments annually, for an average annual rate of 24.3 visits per 100 women between the ages 15 to 44 years. ${ }^{1}$ Acute pelvic pain generally implies pain that is of less than 3 months duration in a toxic, ill appearing and unstable patient, or chronic pain that is worsening., ${ }^{2,3}$ When a female in the reproductive age presents with acute pelvic and/or lower abdominal pain, the first diagnoses to consider are those that are life threatening and would require urgent and/or emergent surgical intervention. ${ }^{4}$ Because the differential diagnosis of acute pelvic pain in the nonpregnant female of reproductive age includes many different organ systems (i.e. gastrointestinal, gynecological, urological, vascular, etc.) a cost-effective and efficient strategy, such as ultrasound, needs to be employed.

It is essential in the assessment of pelvic pain in women of reproductive age to initially exclude pregnancy via urine and/or serum hCG. The diagnosis of pelvic pain is a composite picture (history, physical examination and investigations). No single fact or observation elicits the diagnosis. When evaluating pelvic pain, as with any clinical presentation, it is important to ask about the onset, location, quality, severity, radiation, duration, aggravating and alleviating factors and any temporal changes of the pain overtime. In addition, one must ask about associated symptoms, such as nausea, vomiting, diarrhea, fever, flank pain, dysuria, hematuria, pyuria, frequency, urgency, vaginal bleeding and vaginal discharge. The medical history should focus on the patient's last menstrual period, age of onset of menarche, sexual history, history of sexually transmitted diseases and a complete obstetric history. The physical examination should concentrate on the vital signs, abdominal and pelvic examinations. Unfortunately, in many instances, there is variability of the physical examination (abdominal and pelvic) between examiners. There have been some studies conducted evaluating the validity and reliability of the pelvic examination. ${ }^{5}$ Bimanual examination appears to be of limited value due to a discrepancy in the detection of adnexal masses on physical examination between attending physicians and residents. ${ }^{6}$ Another study conducted in the emergency department setting showed poor interexaminer reliability. ${ }^{7}$

When one considers the organs located in the pelvis and the abdominal cavity whose pain may be referred to the pelvis (i.e. the gall bladder, the appendix, cecum, ascending colon, transverse colon, descending colon, sigmoid, rectum, kidneys, ureter, bladder, uterus, ovaries and fallopian tubes), one has to consider that the diseases most likely originate from these organs. Given such a vast differential diagnosis, the key investigative tool for acute lower abdominal and pelvic pain is the ultrasound..$^{8-15}$ In the emergency department setting ultrasound is the best initial imaging modality for evaluation of pelvic pathology due to its low cost, easy accessibility, widespread availability and lack of ionizing radiation. ${ }^{16}$

Additional investigations include the following:

- CBC: To detect an elevated leukocytosis (nonspecific).

- Urine analysis: To detect a urinary tract infection or hematuria

- Vaginal wet mount: To rule out STD

- Cervical chlamydia and gonorrhea testing: To rule out PID 
Depending on the clinical clues elicited one may categorize the pain as originating from the gastrointestinal, gynecological, and/or urological systems. Ultrasound can be used to evaluate the following differential diagnosis:

- Gastrointestinal sources of both right and left lower quadrant and pelvic pain:

- Appendicitis

- Inflammatory bowel disease

- Inguinal hernia (incarceration and strangulation)

- Diverticulitis

- Irritable bowel syndrome

- Biliary colic

- Cholecystitis

- Gynecological sources of both right and left lower quadrant and pelvic pain:

- Ectopic pregnancy

- Ruptured ovarian cyst

- Adnexal torsion

- Hemorrhagic cyst

- Pelvic inflammatory disease

- Tuboovarian abscess

- Dysmenorrhea

- Endometriosis

- Urological sources of both right and left lower quadrant and pelvic pain:

- Pyelonephritis

- Cystitis

- Urinary retention

- Nephrolithiasis/ureteral stone

- Ureteral obstruction

- Hydronephrosis

\section{CASE REPORTS}

\section{Case 1}

A 20-year-old female (G3 P1021) presents to the emergency department with a 3-day history of fever, abdominal pain, nausea and dysuria. She is currently sexually active and uses oral contraceptive pills only. Onset of menarche was at age 12, first intercourse was at 14 years, with no history of sexually transmitted diseases. Her last menstrual period was 4 weeks ago and was normal. Her medical history includes childhood eczema; normal spontaneous vaginal delivery at age 15 years, two dilations and curettages for elective abortions at ages 17 and 19 years. Her vital signs are blood pressure 110/70 mm Hg, pulse 110 beats per minute, respiratory rate 18 breaths per minute and temperature is $38^{\circ} \mathrm{C}\left(100.4^{\circ} \mathrm{F}\right)$. Her physical examination is significant for bilateral lower quadrant tenderness, whitish yellow vaginal discharge, cervical motion tenderness and bilateral adnexal pain. The remainder of her examination is

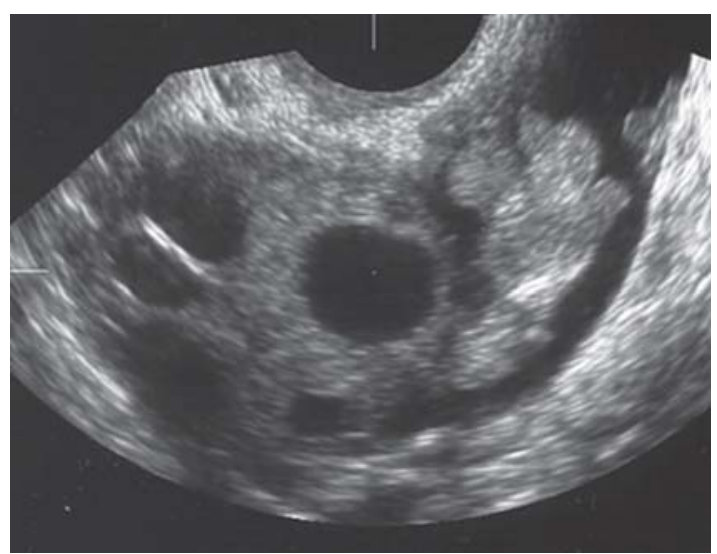

Fig. 1A: Slightly enlarged ovary, thickened walls of the fallopian tube and free fluid in the cul de sac in a patient presenting with acute pelvic pain

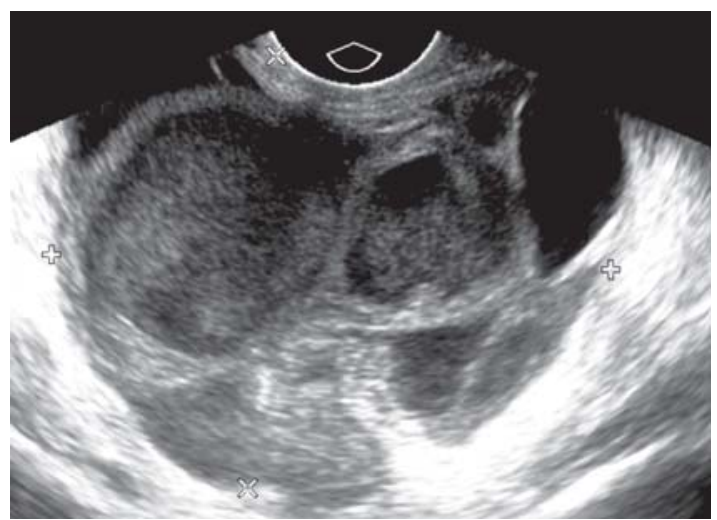

Fig. 1B: Transvaginal scan of a complex adnexal mass with thick septations and echogenic

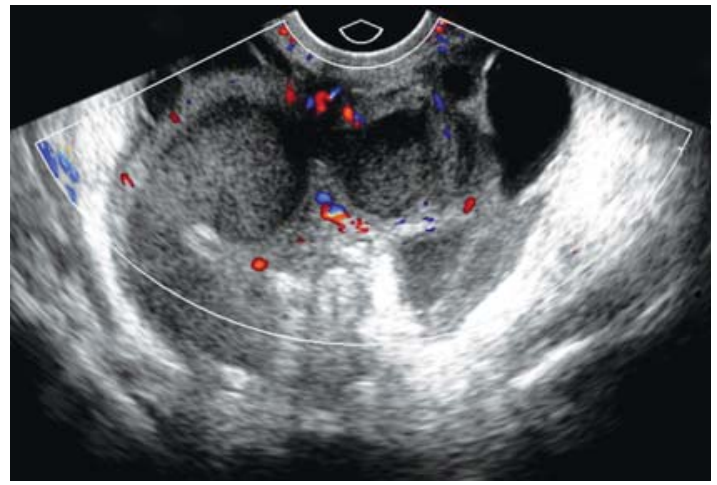

Fig. 1C: The same patient as in Figure 1B. Color Doppler demonstrates increased angiogenesis

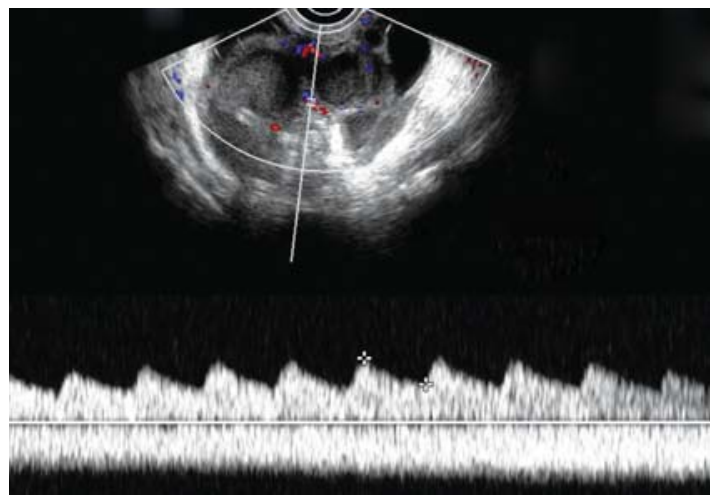

Fig. 1D: Spectral Doppler analysis obtained low impedance blood flow signals with resistive index of 0.41 
normal. Her pregnancy test is negative. Figures $1 \mathrm{~A}$ to D demonstrate her pelvic ultrasound findings.

\section{Case 2}

A 38-year-old female (G0P0) presents to your office with a 1-day history of sharp right lower quadrant pain. The pain started this morning and has progressively worsened. She also complains of being nauseated and has vomited twice. She is currently sexually active and is undergoing preparation for in vitro fertilization. Onset of menarche was at age 13, age of first intercourse was 22 years, with no history of sexually transmitted diseases. Her medical history is unremarkable. Her medications include oral contraceptives (discontinued), and GnRH analog, leuprolide. Her vital signs are blood pressure 115/80 mm Hg, pulse 110 beats per minute, respiratory rate 20 breaths per minute and temperature is $37^{\circ} \mathrm{C}\left(98.6^{\circ} \mathrm{F}\right)$. Her physical examination is significant for right lower quadrant tenderness, right adnexal enlargement and tenderness. The remainder of her

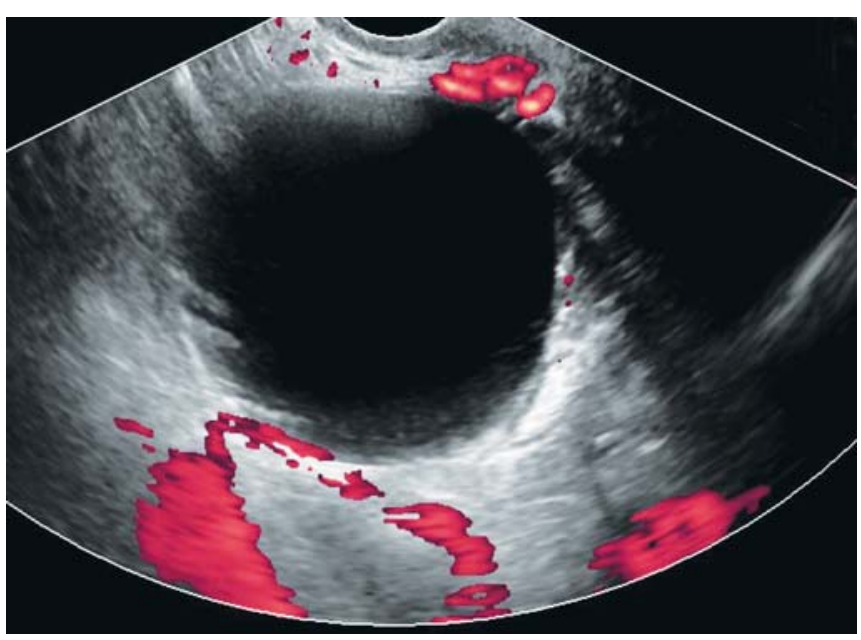

Fig. 2A: Simple ovarian cyst. Power Doppler displays ovarian blood flow signals, virtually excluding adnexal torsion

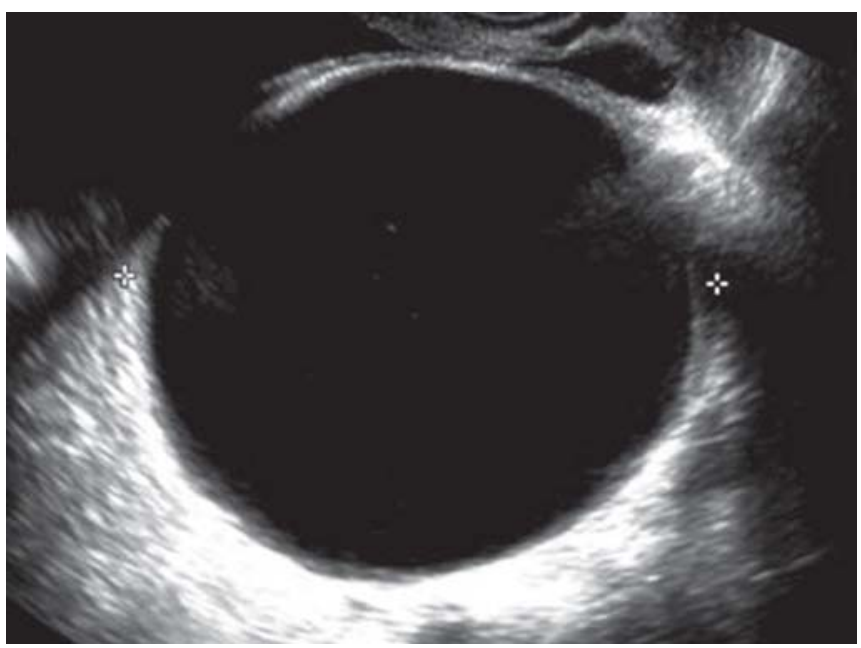

Fig. 2B: Power Doppler scan of another patient presenting with acute pelvic pain. Note the complete absence of ovarian blood flow signals examination is normal. Her urine pregnancy test is negative. Figures 2A and B illustrate her B mode and color Doppler ultrasound findings.

\section{Case 3}

A 30-year-old female (G1 P1001) presents to the emergency department with a sudden onset of right lower quadrant pain. The pain started after her usual morning exercise routine. There is no vaginal bleeding or discharge. She is currently sexually active, in a monogamous relationship with her husband and wants to become pregnant. Her last menstrual period was 3 weeks ago and was normal. Onset of menarche was at age 14, age of her first intercourse was 17 years, with no history of sexually transmitted diseases. She had a normal spontaneous vaginal delivery 3 years ago. She currently is not on any medications and discontinued her oral contraceptive pills 4 months ago. Her urine pregnancy test is negative. Her vital signs are normal. Her physical examination is significant for right lower quadrant tenderness, guarding and rebound. Her pelvic examination is significant for right adnexal tenderness, and no masses appreciated. The remainder of her examination is normal. Figure 3 illustrates her pelvic ultrasound results.

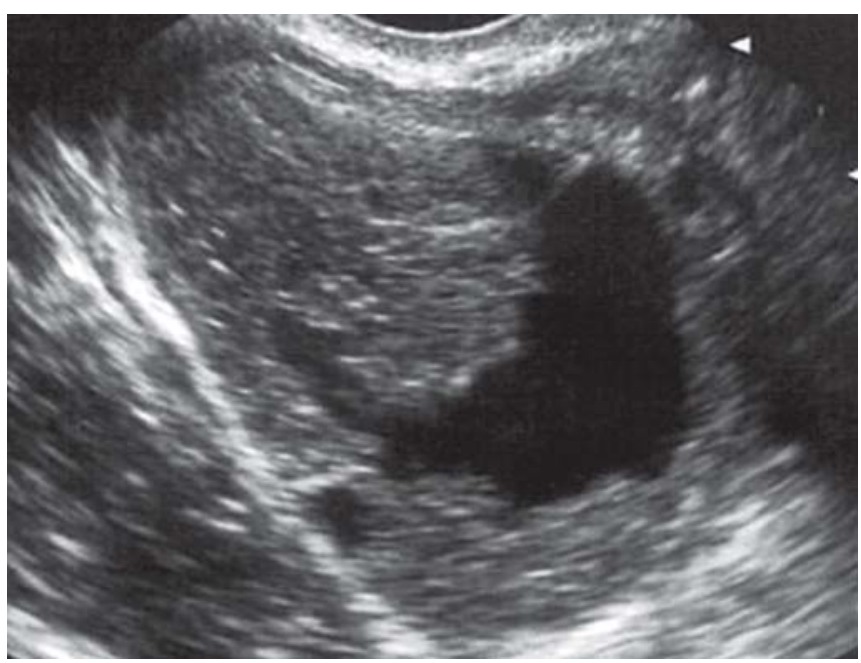

Fig. 3: Ruptured ovarian cyst in a nonpregnant patient presenting with sudden onset of right low quadrant pain. Note the irregular contours of the ovarian cystic lesion

\section{Case 4}

A 32-year-old female (G0P0) new to your practice presents to your office with a 1-week history of dull right-sided abdominal and pelvic pain. She has no other complaints. She is currently not sexually active. Onset of menarche was at age 14, age of first intercourse was 17 years, with no history of sexually transmitted diseases. Her last menstrual period was 10 days ago and she had greater than 




Fig. 4: Color Doppler image of a complex ovarian lesion in a 32 year-old patient presenting with right low quadrant pain. Note echogenic fluid and thin septations. Color Doppler demonstrates regularly separated peripheral vessels

normal bleeding. She has a history of painful heavy periods. She was told that 'she may have an ovarian cyst' by her primary care physician 2 years ago but has had no workup. Her vital signs are blood pressure 120/80 mm Hg, pulse 105 beats per minute, respiratory rate 20 breaths per minute and temperature is normal. Her urine pregnancy test is negative. Her examination is significant for right costovertebral angle, right lower quadrant and suprapubic tenderness. Her pelvic examination is significant for blood in the cervical canal and right adnexal tenderness. Her pelvic ultrasound is seen in Figure 4.

\section{Case 5}

A 22-year-old female (G0P0) presents to the emergency department with 6 hours of abdominal pain. The pain is currently in the right lower quadrant, associated with nausea and several episodes of vomiting. She has no dysuria, hematuria, vaginal bleeding or discharge. She is currently sexually active and is using no form of contraception. Her last menstrual period was 6 weeks ago and she is 2 weeks late. Onset of menarche was at age 10, age of first intercourse was 13 years, with no history of sexually transmitted diseases. Her urine pregnancy test is negative. Her medical history is significant for ovarian cysts. Her vital signs are blood pressure 108/75 mm Hg, pulse 120 beats per minute, respiratory rate 22 breaths per minute and temperature is $39^{\circ} \mathrm{C}\left(102.2^{\circ} \mathrm{F}\right)$. Her physical examination is significant for right lower quadrant tenderness guarding and rebound. Her pelvic examination reveals mild right adnexal tenderness with no adnexal enlargement, and no cervical motion tenderness. The remainder of her examination is normal. Pelvic ultrasound does not visualize presence of ovarian

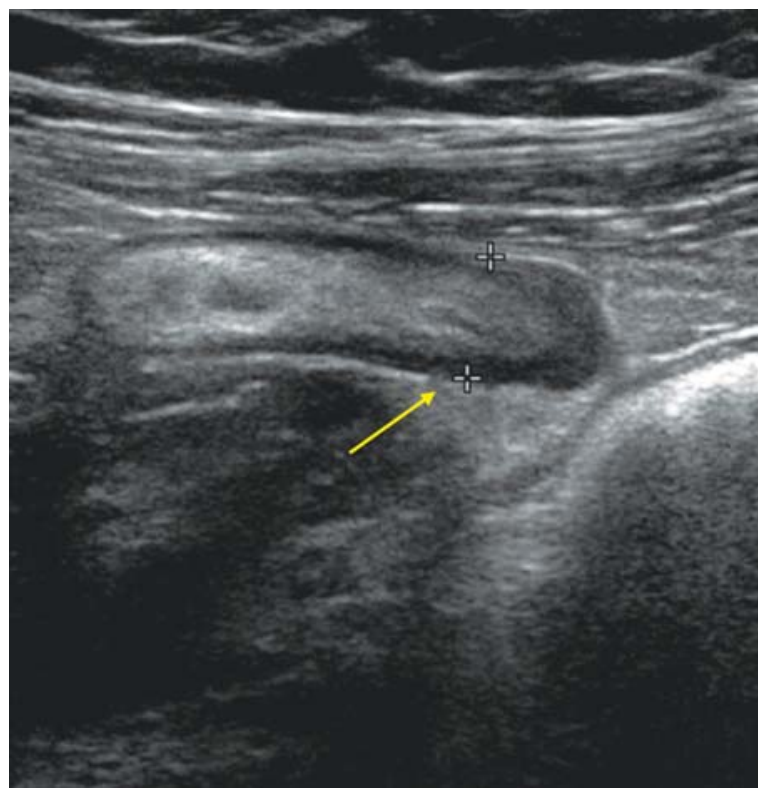

Fig. 5A: Ultrasound image of right low abdominal quadrant. Note a tubular noncompressible structure measuring $9 \mathrm{~mm}$ (arrow)

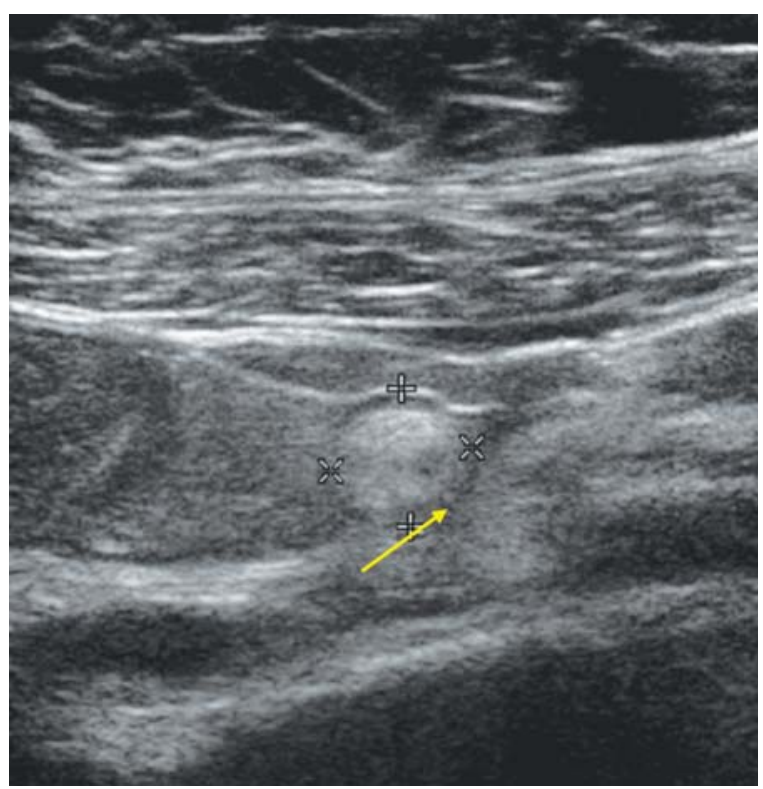

Fig. 5B: The same structure demonstrated in transverse section (arrow)

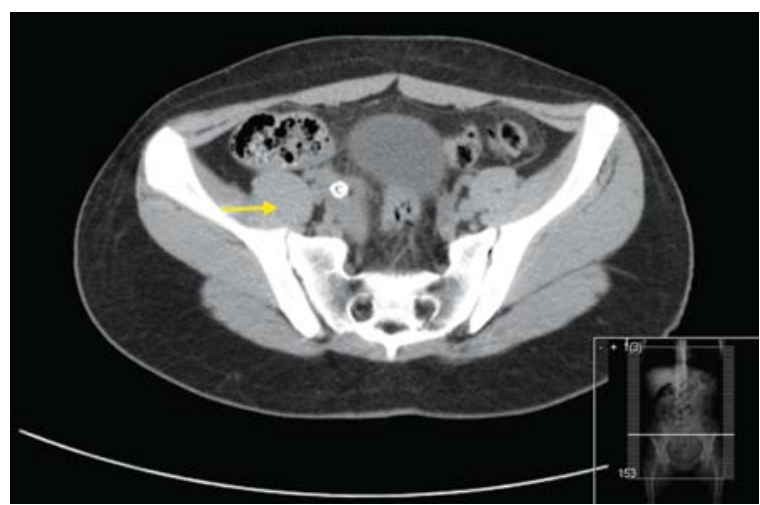

Fig. 5C: Abdominal CT of the same patient (arrow) 
cysts. Figures 5A to C demonstrate her ultrasound and CT images.

\section{Case 6}

A 37-year-old female (G3 P3003) presents to the emergency department with a chief complaint of sharp right back, right lower quadrant and right pelvic pain. When she went to the bathroom she noticed blood in the toilet. She denies dysuria, fever or chills. She is currently sexually active, in a monogamous relationship and is using oral contraceptives pills only. Onset of menarche was at age 12, age of first intercourse was 24 years with no history of sexually transmitted diseases. She has had 3 normal spontaneous vaginal deliveries at ages 25 years, 27 years and 31 years. Her last menstrual period was 4 weeks ago and was normal. Her urine pregnancy test is negative. Her vital signs are significant for mild tachycardia and are otherwise normal.

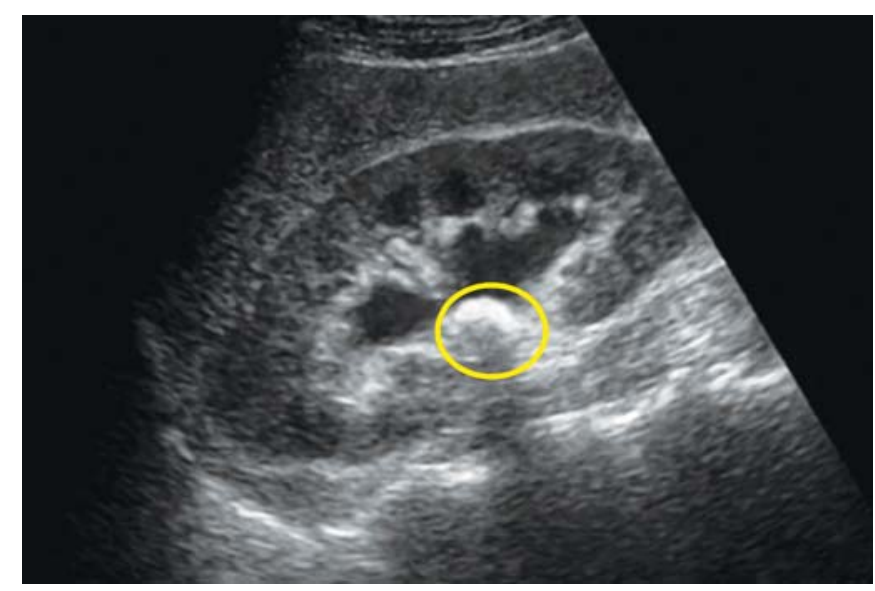

Fig. 6A: Renal ultrasound of a patient with right lower pelvic pain and right flank pain. Note dilated pelvicalyceal system (hydronephrosis) and a $1 \mathrm{~cm}$ hyperechogenic area in the renal pelvis (yellow circle)

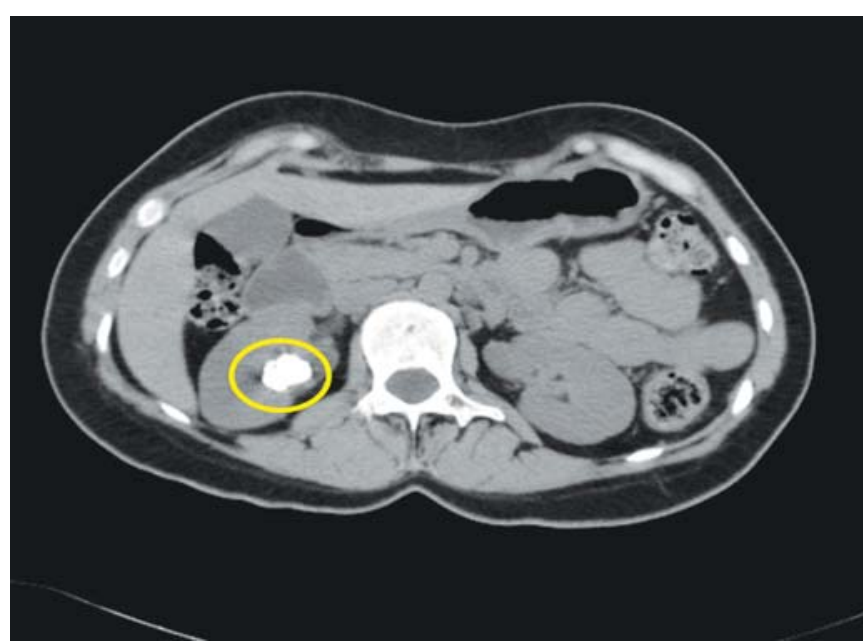

Fig. 6B: Axial CT scan of the same patient. Yellow circle corresponds to the area demonstrated on ultrasound. In this section, hydronephrosis was not visualized
Her physical examination is significant for right costovertebral angle and right lower quadrant tenderness. The remainder of her examination including pelvic examination is normal. Figures $6 \mathrm{~A}$ and $\mathrm{B}$ demonstrate her ultrasound and CT findings.

\section{Case 7}

A 22-year-old female (G3P0030) presents to your office with a 3-day history of suprapubic pain. She also complains of nausea without vomiting, dysuria and frequency, and denies any fever, chills, vaginal bleeding and vaginal discharge. She is currently sexually active and uses no contraception. Onset of menarche was at age 13 years, age of first intercourse was 15 years. Her last menstrual period was 4 weeks ago and was normal. Her medical history includes a history of Chlamydia at age 17; 3 dilations and curettages for elective abortions at ages 16, 17 and 20 respectfully. Her vital signs are normal and her physical

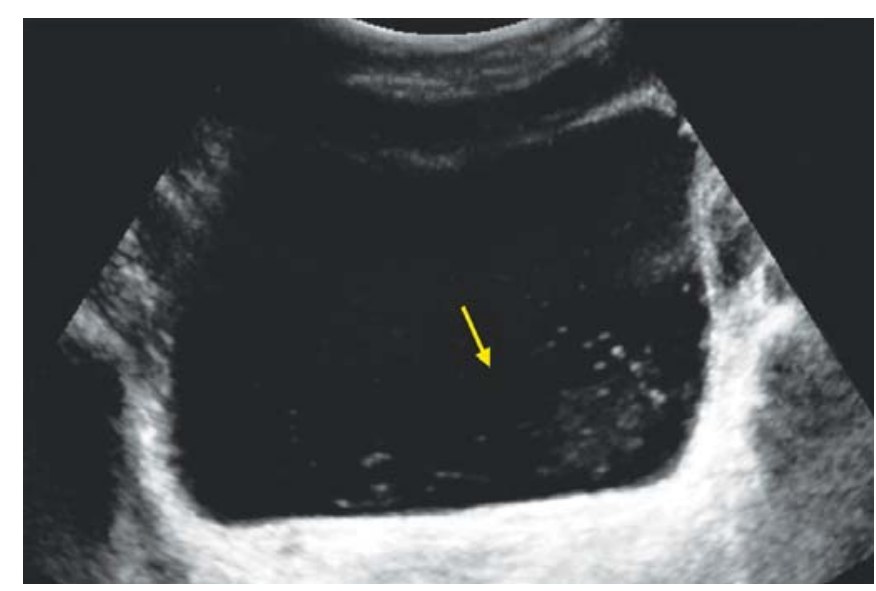

Fig. 7A: Ultrasound images of the urinary bladder in a 22-year-old female with burning micturition and urgency. Urinary bladder is filled with sludge and mobile internal echoes (yellow arrow)

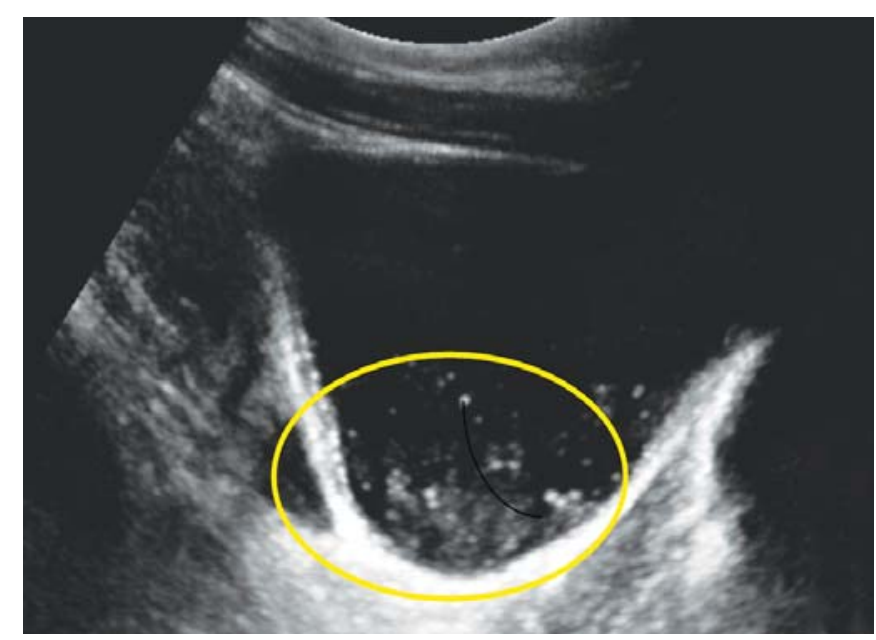

Fig. 7B: Sonographic findings of thickened wall of the urinary bladder and mobile debris (yellow circle) are suggestive of cystitis complicated with pyuria or hematuria 
examination is significant for suprapubic and uterine tenderness. Her urine pregnancy test is negative. Figures 7A and $\mathrm{B}$ reveal her ultrasound findings.

\section{Case 8}

A 39-year-old female (G4P4004) presents to your office with a 5-day history of sharp right flank, right upper and right lower quadrant pain. The pain started 5 days ago and has progressively worsened. She does state that, for the past 2 to 3 years, she occasionally would get 'shooting pains' in her epigastrium and right upper quadrant after meals but attributed it to 'gas'. She also complains of some nausea, but no vomiting. She denies dysuria, hematuria, vaginal bleeding or any abnormal vaginal discharge. She is currently sexually active in a monogamous relationship with her husband. She has had four normal spontaneous vaginal deliveries at ages 26, 28, 30 and 32 years. Onset of menarche was at age 12, age of first intercourse (coitarche) was 14 years, with no history of sexually transmitted diseases. Her medical history is significant for asthma. Her medications include oral contraceptives. Her vital signs are normal. Her physical examination is significant for right costovertebral angle tenderness, right upper quadrant tenderness and right lower quadrant tenderness. There is no guarding or rebound. Her pelvic examination is unremarkable. The remainder of her examination is normal. Her urine pregnancy test is negative.

\section{CASE DISCUSSION}

\section{Case 1: Pelvic Inflammatory Disease}

Pelvic inflammatory disease refers to the infection of the upper female genital tract (uterus, fallopian tubes, oviducts and ovaries) caused by ascending spread of bacteria from the vagina or cervix. ${ }^{17}$ Classically, the patient is a

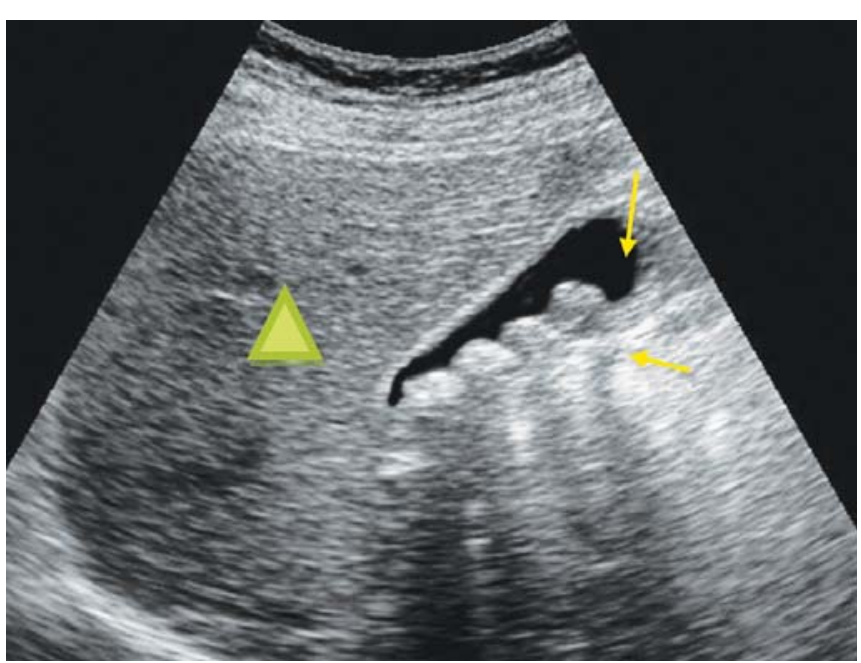

Fig. 8: Ultrasound of the right upper quadrant in a patient with RUQ abdominal pain. Gallbladder is pointed by red arrow. Green triangle indicates liver. Gallstones layered along the wall of gallbladder are shown by yellow arrow menstruating female who has had multiple sexual partners and does not use barrier contraception and complains of pelvic pain and/or lower abdominal pain with vaginal discharge. The patient in case 1 is a 20-year-old (G3 P1021) female who presents with fever, lower abdominal pain, with early onset of first coitus, and multiple sexual partners. Her physical examination reveals bilateral lower quadrant tenderness, with vaginal discharge, cervical motion tenderness and bilateral adnexal tenderness. In a patient with acute pelvic pain, early sonographic signs of salpingoophoritis include enlarged ovaries, hyperemia, swelling and tortuosity of the fallopian tubes, free fluid in the cul-desac, purulent exudate (originating from the tubal lumen and serosal surfaces of the tubes). ${ }^{17}$ Uterine findings include mild-to-moderate uterine enlargement, increased endometrial thickness ( $>12 \mathrm{~mm}$ ) and altered echogenicity of the endometrium. ${ }^{17}$ Color Doppler demonstrates increased angiogenesis as seen in Figure $1 C .{ }^{18}$ If untreated, patients may develop a tubo-ovarian abscess. A complex adnexal mass with thick septations and echogenic cysts is suggestive of a tubo-ovarian abscess. ${ }^{18}$ The most common organisms associated with acute PID are Neisseria gonorrhoeae and Chlamydia trachomatis. ${ }^{19}$ Antimicrobial treatment should be directed at the most prevalent organisms. In addition, the patient needs to be counselled regarding methods of preventing PID and sexuallty transmitted infections.

\section{Case 2: Adnexal Torsion}

Adnexal torsion refers to torsion of the ovaries, the fallopian tubes or both. ${ }^{20}$ Clinically, the patient presents with sudden onset of unilateral abdominal and/or pelvic pain. Other nonspecific symptoms include nausea, vomiting and, in some patients, fever. ${ }^{21}$ The primary pathology invariably involves an enlarged ovary. This enlarged ovary alters the position of the fallopian tube setting up the scenario for torsion. Torsions lead to strangulation compromising blood flow, initially venous, then arterial blood flow, ultimately leading to ischemia and necrosis. ${ }^{20}$ With early diagnosis, the prognosis is excellent. Delay in diagnosis may affect fertility in certain populations. The patient in case 2 is a 38-yearold (G0P0) female who presents with a 1 day history of sharp right lower quadrant pain. She is undergoing treatment for infertility and has taken oral contraceptives and leuprolide. Physical examination reveals right lower quadrant and right adnexal tenderness. Her pregnancy test is negative. At this point appendicitis and ovarian torsion are the leading differential diagnosis. As stated in the introduction, ultrasound has the advantage of having high specificity for detection of pelvic pathology thus initially 
avoiding radiation. Ultrasound may also be used to view the appendix. If no pathology is detected one can then order a CT scan of the abdomen and pelvis. Her ultrasound reveals a unilocular sonolucent cystic structure with no evidence of ovarian blood flow signals (Fig. 2B). In most cases of adnexal torsion a pelvic mass is detected..$^{22}$ Other findings include reduced and/or absent blood flow. If there is a high suspicion of adnexal torsion, urgent laparoscopic intervention is indicated. ${ }^{23,24}$

\section{Case 3: Rupture of an Ovarian Cyst}

Any collection of fluid surrounded by a thin wall within the ovary exceeding 2.5 to $3 \mathrm{~cm}$ in mean diameter is termed an ovarian cyst. Ovarian cysts are extremely prevalent. ${ }^{25}$ The majority of ovarian cysts are benign. ${ }^{25}$ Rupture of an ovarian cyst commonly presents as sudden onset of lower abdominal and/or pelvic pain. This presentation may be similar to an ovarian torsion and/or a hemorrhagic cyst. In addition, due to peritoneal irritation, patients may present with nausea and/or vomiting. The patient in case 3 is a 30year-old female (G1 P1001) who presents to the emergency department with sudden onset of right lower quadrant pain. Her physical examination reveals right lower quadrant tenderness, guarding and rebound. Her pelvic examination is significant for right adnexal tenderness. As with adnexal torsion, the initial imaging modality is ultrasound. Her pelvic ultrasound detected a cystic structure with irregular borders. Other ultrasonographic findings include free peritoneal fluid. ${ }^{25}$ Ruptured ovarian cysts are usually managed expectantly with analgesia and observation. ${ }^{25}$

\section{Case 4: Hemorrhagic Ovarian Cyst}

A hemorrhagic ovarian cyst is a cyst that is filled with blood, which usually occurs when a blood vessel breaks into the cyst. Pain from a hemorrhagic cyst is probably due to stretching of the ovarian capsule, as opposed to pain from ovarian cyst rupture which is due to peritoneal irritation. ${ }^{25}$ Similar to ovarian torsion and ruptured ovarian cysts, patients with a hemorrhagic cyst also present with unilateral lower abdominal and/or pelvic pain associated with nausea, vomiting and/or vaginal bleeding. The patient in case 4 is a 32-year-old female (G0P0) with a 1-week history of dull right sided abdominal and pelvic pain. She has no other complaints. Her examination is significant for right costovertebral angle tenderness and right lower quadrant and suprapubic pain. Her pelvic examination is significant for blood and right adnexal tenderness. Again, the differential diagnosis is varied. As with both adnexal torsion and ruptured ovarian cyst, ultrasound is the initial imaging investigation of choice: Low cost, easy accessibility and no radiation exposure. ${ }^{25}$ Because hemorrhagic cysts evolve in different stages, fresh blood on the ultrasound initially appears anechoic, later transforming to a blood clot (echogenic content with thin septations) and finally resolving. ${ }^{25,26}$ The echogenicity of the hemorrhagic cyst diminishes as the red blood cell undergoes hemolysis. ${ }^{26}$ The typical appearance on ultrasound is that of an enlarged ovary containing multiple echogenic areas (representing blood clots) in a reticular pattern due to clot (representing fibrin strands, not tissue septations) retraction. ${ }^{25}$ Her ultrasound revealed a complex ovarian lesion with numerous thin septations. Color Doppler reveals peripheral vascularity (Fig. 4). Pulsed Doppler waveform analysis demonstrates low to moderate impedance to blood flow signals, typical for luteal conversion. A hemorrhagic cyst also has the potential to rupture. As with a uncomplicated simple ovarian and ruptured cyst, the treatment is expectant with an ultrasound follow-up in 6 weeks. ${ }^{26}$ Occasionally, patients present with sudden pain and profuse intraabdominal bleeding, requiring laparoscopic surgery.

\section{Case 5: Acute Appendicitis}

Appendicitis occurs when there is a progressive increase in intraluminal pressure that occurs when the appendix is blocked (i.e. fecalith), which leads to a compromise in venous outflow, ischemic injury, stasis of luminal contents which progresses to inflammation, infection, necrosis and finally perforation. Classically, appendicitis begins with nonspecific periumbilical pain which over the course of the next 24 hours localizes to the right lower quadrant. In addition, the pain may radiate to the right flank and right pelvic area. The patient may also present with nausea, vomiting, fever and a decreased appetite. The symptoms are very similar to the cases described above and listed in the differential diagnosis. Unfortunately, the current role of ultrasound for the diagnosis of appendicitis is limited in the United States. ${ }^{27} \mathrm{CT}$ is currently the diagnostic investigation of choice. ${ }^{27}$ Ultrasound offers widespread availability, low cost and absence of ionizing radiation. ${ }^{27} \mathrm{In}$ addition, it is the initial imaging modality for almost all pelvic pathologies. The patient in case 6 is a 22-year-old female (G0P0) who presents to the emergency department with 6 hours of abdominal pain. The pain is currently in the right lower quadrant associated with nausea and several episodes of vomiting. Her medical history is significant for ovarian cysts. One can order a CT scan of the abdomen and pelvis and not be faulted, but would be exposing a patient to radiation. An alternative is to order an ultrasound, view the appendix and look for other causes of pelvic pain. The results of her ultrasound revealed a tubular noncompressible 
structure measuring $9 \mathrm{~mm}$ suggestive of appendicitis, which was confirmed by CT imaging (Figs 5A to C). The current standard of care for appendicitis is an appendectomy.

\section{Case 6: Ureterolithiasis}

Nephrolithiasis refers to stones in the kidneys. Ureteral calculi or ureterolithiasis refers to stones in the ureters. Almost all ureteral calculi originate in the kidneys. The classic presentation is flank pain, hematuria, nausea and vomiting. In addition, the pain may radiate to the lower quadrant and pelvic area. This is a common clinical presentation to emergency departments. ${ }^{28}$ Current standard of care is nonenhanced CT scan of the abdomen and pelvis. There has been increasing concern regarding the exposure to radiation. Recently there have been studies to show the efficacy of ultrasound for detection of calculi and secondary signs of hydronephrosis. ${ }^{28}$ In addition, as mentioned earlier, ultrasound has the advantage of detecting other pelvic pathologies. The patient in case 6 is a 37-year-old female (G3 P3003) with a chief complaint of sharp right back, right lower quadrant and right pelvic pain. Her physical examination is significant for right costovertebral angle and right lower quadrant tenderness. An ultrasound was ordered and her results revealed a $1 \mathrm{~cm}$ stone in the renal pelvis, causing dilatation of the pelvicalyceal system (Fig. 6A). Sonographic finding was confirmed by CT imaging (Fig. 6B). Treatment depends on the patients' clinical presentation and stone size. Most uncomplicated small ureteral stones (less than $5 \mathrm{~mm}$ ) with mild hydronephrosis are managed with observation and analgesia. Generally, stones greater than $5 \mathrm{~mm}$ will require consultation with urologist.

\section{Case 7: Acute Cystitis}

Urinary tract infections encompass both the lower (cystitis) and upper urinary tracts (pyelonephritis), and accounts for over 7 million office and 1 million emergency department visits. ${ }^{29}$ Approximately 24 to $40 \%$ of women in the United States between the ages 20 to 40 have had urinary tract infections. ${ }^{30}$ The main organisms responsible for both upper and lower urinary tract infections are E. coli. Other organisms include Proteus species, S. saprophyticus, Klebsiella species and Enterococcus faecalis. Classically, patients present with dysuria, urgency, frequency, lower abdominal and/or pelvic discomfort. The pain may be referred to the right and left lower quadrants and flanks. Many patients may have suprapubic tenderness and/or costovertebral tenderness. In most cases, the diagnosis is based on clinical features along with urine analysis and cultures. These symptoms are very similar to all the previous cases. Pelvic inflammatory disease, vaginitis or cervicitis may also cause dysuria. The patient in case 7 is a 22-yearold (G3P0030) female who presents with a 3-day history of suprapubic pain, nausea and dysuria. She is currently sexually active and uses no contraception. Her medical history includes a history of Chlamydia at age 17; three dilations and curettages for elective abortions at ages 16 , 17 and 20 respectively. Her physical examination is significant for suprapubic and uterine tenderness. Given her history, the differential diagnosis needs to be expanded. Again, ultrasound has the advantage of evaluating the spectrum of pelvic pathologies. A transabdominal ultrasound for this case revealed a urinary bladder filled with sludge and mobile echoes (Figs 7A and B). Antimicrobial treatment is directed to the most prevalent microorganisms.

\section{Case 8: Cholelithiasis}

It is estimated that cholelithiasis and/or gallstones affect 10 to $12 \%$ of the adult population in developing countries. ${ }^{31}$ Gallstones may be present for years without causing any pain. Classically, the patient presents with spasmodic or 'colicky' pain that begins in the epigastrium or right upper quadrant after a fatty meal. If the patient has fever, chills and right upper quadrant tenderness the patient may have acute cholecystis. In either case, the pain may radiate to the flanks or right lower quadrant. The patient in case 8 is a 39-year-old female (G4 P4004) who presents with a 5-day history of sharp right flank, right upper and right lower quadrant pain. For the past 2 to 3 years, after meals, she occasionally experiences 'shooting pains' in her epigastrium and right upper quadrant. Her physical examination is significant for right costovertebral angle tenderness, right upper quadrant tenderness and right lower quadrant tenderness. Her history suggests that the pain originates from the gallbladder, but her physical examination requires the clinician to expand the differential diagnosis. Ultrasound is the initial diagnostic imaging modality for the detection of gallbladder and/or biliary tract disease. As illustrated in the previous cases, it is relatively inexpensive, easily accessible and does not involve any radiation. In addition, if one does not diagnose any gallbladder pathology; one has the capability of viewing other organ systems. Gallstones appear as echogenic foci that cast an acoustic shadow. The features of acute cholecystitis on an ultrasound include a sonographic Murphy sign, visualization of gallbladder wall thickening, pericholecystic fluid and gallbladder distension. Her ultrasound revealed gallstones layered along the wall of gallbladder (Fig. 8). Treatment is dependent on the patient's clinical presentation and duration of the disease. 
Asymptomatic gallstones may be managed expectantly, but once frequent and severe episodes of pain occur, surgical consultation is indicated for a possible cholecystectomy.

\section{REFERENCES}

1. Curtis K, Hillis S, Kieke BJ, Brett K, Marchbanks P, Peterson $\mathrm{H}$. Visits to emergency departments for gynecologic disorders in the United States 1992-1994. Obstet Gynecol 1998;91(6):1007-12.

2. Karnath BM, Breitkopf DM. Acute and chronic pelvic pain in women. Hospital Physician. July 2007:41-48.

3. Penner R, Majumdar S. 'Diagnostic approach to abdominal pain in adults’ 2011:1-19. http://www.uptodate.com (10/25/2011).

4. Jung PJ, Merrell RC. Acute abdomen. Gastroenterol Clin North Am 1988:17:227.

5. Kruszka PS, Kruszka SJ. Evaluation of acute pelvic pain in women. American Family Physician 2010;82(2):141-47.

6. Padilla LA, Radosevich DM, Milad MP. Accuracy of the pelvic examination in detecting adnexal masses. Obstet Gynecol 2000;96(4):593-98.

7. Close RJ, Sachs CJ, Dyne PL. Reliability of bimanual pelvic examinations performed in emergency departments. West J Med 2001;175(4):240-45.

8. Vandermeer FQ, Wong-You-Cheong JJ. Imaging of acute pelvic pain. Clin Obstet Gynecol 2009;52(1):2-20.

9. Laméris W, van Randen A, van Es HW, et al. OPTIMA study group. Imaging strategies for detection of urgent conditions in patients with acute abdominal pain: Diagnostic accuracy study. BMJ 2009;338:b2431.

10. Yamamoto W, Kono H, Maekawa M, Fukui T. The relationship between abdominal pain regions and specific diseases: An epidemiologic approach to clinical practice. J Epidemiol 1997;7:27.

11. Heikkinen M, Pikkarainen P, Eskelinen M, Julkunen R. GPs' ability to diagnose dyspepsia based only on physical examination and patient history. Scand J Prim Health Care 2000; 18:99.

12. Thomson AB, Barkun AN, Armstrong D, et al. The prevalence of clinically significant endoscopic findings in primary care patients with uninvestigated dyspepsia: The Canadian adult dyspepsia empiric treatment—prompt endoscopy (CADET-PE) study. Aliment Pharmacol Ther 2003; 17:1481.

13. Böhner H, Yang Q, Franke C, et al. Simple data from history and physical examination help to exclude bowel obstruction and to avoid radiographic studies in patients with acute abdominal pain. Eur J Surg 1998; 164:777.

14. Eskelinen M, Ikonen J, Lipponen P. Usefulness of history-taking, physical examination and diagnostic scoring in acute renal colic. Eur Urol 1998; 34:467.

15. Trowbridge RL, Rutkowski NK, Shojania KG. Does this patient have acute cholecystitis? JAMA 2003; 289:80.

16. Yitta S, Mausner VE, Kim A, et al. Pelvic ultrasound immediately following MDCT in female patients with abdominal/pelvic pain: Is it always necessary? Emerg Radiol 2011;18:371-80.
17. Kupesic S, Kurjak A, Baston K. Color Doppler, 3D and 4D ultrasound in gynecology, infertility and obstetrics (2nd ed). New Delhi, India. Jaypee Brothers Medical Publishers 2011;Chapter 7:89-95.

18. Kupesic S, Kurjak A, Pasalic L, Benic S, Ilijas M. The value of transvaginal color Doppler in the assessment of pelvic inflammatory disease. Ultrasound in Medicine and Biology 1995;21(6):733-38.

19. Bakken IJ, Ghaderi S. Incidence of pelvic inflammatory disease in a large cohort of women tested for Chlamydia trachomatis: A historical follow-up study. BMC Infect Dis 14 Aug 2009;9(1):130.

20. Oelsner G, Shashar D. Adnexal torsion. Clinical Obstetrics and Gynecology 2006;49(3):459-63.

21. Houry D, Abbott JT. Ovarian torsion: A fifteen-year review. Ann Emerg Med 2001;38:156-59.

22. Breech LL, Hillard PJ. Adnexal torsion in pediatric and adolescent girls. Curr Opin Obstet Gynecol 2005;17:483-89.

23. White M, Stella J. Ovarian torsion: 10-year perspective. Emerg Med Australias 2005;17:231-37.

24. Kupesic S, Plavsic BM. Adnexal torsion: Color Doppler and three-dimensional ultrasound. Abdominal Imaging 2010;35: 602-06.

25. Bottomley C, Tom B. Diagnosis and management of ovarian cyst accidents. Best Practice and Research Clinical Obstetrics and Gynaecol 2009;23:711-24.

26. Jain KA. Sonographic spectrum of hemorrhagic ovarian cysts. Journal Ultrsound Medicine 2002;21:879-86.

27. Maturen KE, Wasnik AP, Kamaya A, et al. Ultrasound imaging of bowel pathology: Technique and keys to diagnosis the acute abdomen. AJR 2011;197:1067-75.

28. Tamm EP, Silverman PM, Shuman WP. Evaluation of the patient with flank pain and possible ureteral calculus. Radiology 2003;228:319-29.

29. Craig WD, Wagner BF, Travis MD. Pyelonephritis: Radiologicpathologic review. Radiographics 2008;28:255-76.

30. Brusch JL. Urinary tract infection in females. Medscape Reference Drugs, Diseases and Procedures 2011(Aug):1-9.

31. Crawford James M. Robbins and Coltran pathologic basis of disease (7th ed): Elsevier Saunders 2004; Chapter 18:877-937.

\section{ABOUT THE AUTHORS}

\section{Nadah Zafar}

Assistant Professor, Department of Medical Education, Paul L Foster School of Medicine, Texas Tech University, El Paso, Texas, USA

\section{Sanja Kupesic Plavsic (Corresponding Author)}

Professor, Department of Obstetrics and Gynecology, Paul L Foster School of Medicine, Texas Tech University, 4800 Alameda Ave, El Paso, TX 79905, Texas, USA, Phone: +1 (915)783-1700 e-mail: sanja.kupesic@ttuhsc.edu 


\section{SELF-ASSESSMENT QUIZ}

1. A 32-year-old woman presents to the emergency department with right sided abdominal pain, nausea and vomiting. She has had intermittent episodes for the past 1 year usually after eating a heavy meal but it would resolve. In the past couple of days her symptoms have been getting worse, and now she presents with fever. She has no medical history. Her last normal menstrual period was 2 weeks age. On examination, she has a temperature of $39^{\circ} \mathrm{C}$, her pulse rate is 110 , her respiratory rate is 20 and her blood pressure is $110 / 80$. Her abdominal examination is significant for right upper quadrant tenderness. Her heart and lung examinations are normal. Her pregnancy test is negative. Given her clinical presentation what is the best initial investigation for the diagnosis you are considering?
A. Complete blood count
B. Transvaginal ultrasound
C. CT scan of the abdomen and pelvis
D. Ultrasound of the right upper quadrant

2. A 30-year-old female present to the emergency department with periumbilical pain radiating to the epigastric area which started the night before. It is associated with nausea and two episode of vomiting and one episode of loose bowel movement. Her menstrual period is late by 1 week. She has a past medical history for an ectopic pregnancy. On examination, her blood pressure was 106/80, pulse rate was 102 temperature was $37.5^{\circ} \mathrm{C}$, and respiratory rate was 18 . Her heart and lung examination were normal. Her abdominal examination showed mildly distended abdomen, with no upper quadrant or epigastric tenderness, she had severe right lower quadrant tenderness. Her pelvic examination was normal. What is the next initial/immediate investigation?
A. CT scan of the abdomen and pelvis with intravenous contrast
B. Ultrasound of the pelvis
C. Complete blood count
D. Serum beta hCG test

3. A 22-year-old female presents to the emergency department with periumbilical /epigastric pain that started a couple of hours ago. It was associated with nausea, vomiting and three episodes of loose bowel movement. Her last normal menstrual period was 1 week ago and she is not sexually active. She has no medical or surgical history. On examination, she has a temperature of $38.5^{\circ} \mathrm{C}$, pulse rate of 106 , respiratory rate of 18 breaths per minute, and blood pressure of 121 / $76 \mathrm{~mm} \mathrm{Hg}$. Her heart and lung examination were normal. Her abdominal examination was significant for right lower quadrant tenderness. She had no epigastric or upper quadrant tenderness. Her serum pregnancy test was negative, urine analysis and complete blood count demonstrated leukocytosis. Her pelvic examination was normal. Given the clinical scenario, what is the most likely etiology of her symptoms?
A. Ruptured ovarian cyst
B. Ureterolithiasis
C. Acute appendicitis
D. Pelvic inflammatory disease

4. The following is an ultrasound of a 29-year-old female who presents with sudden onset of right lower quadrant pain, based on this image what is the most likely diagnosis?

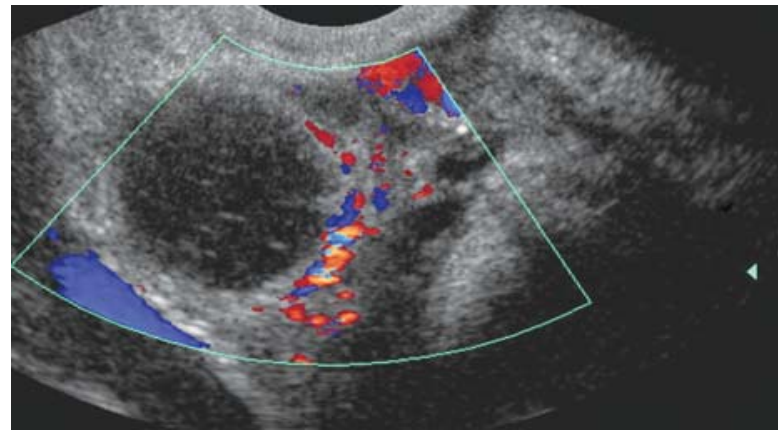



A. Salpingo-oophoritis
B. Ovarian cyst
C. Hemorrhagic cyst
D. Ovarian torsion

5. A 20-year-old female who has a history of pelvic inflammatory disease in the past presents with a 1-day history of left abdominal and pelvic pain, and dysuria. On physical examination she has left flank, left lower quadrant and suprapubic tenderness. Her serum beta hCG is negative. Based on her clinical presentation what is the most likely diagnosis?
A. Pelvic inflammatory disease
B. Ovarian cyst
C. Pyelonephritis
D. Tubo-ovarian abscess

6. A 17-year-old female with high-risk sexual behavior presents with a 5-day history of fever, bilateral lower abdominal and pelvic pain and tenderness. Based on the clinical information, what is the most likely diagnosis?
A. Ruptured ovarian cyst
B. Tubo-ovarian abscess
C. Acute appendicitis
D. Hemorrhagic cyst

7. A 34-year-old female presents with sudden onset of left-sided abdominal pain, nausea and vomiting. Her serum beta hCG is negative. Her pain started after she came back from exercising. Based on the clinical information, what is the most likely diagnosis?
A. Adnexal torsion
B. Tubo-ovarian abscess
C. Ruptured ovarian cyst
D. Hemorrhagic cyst

8. A 31-year-old female with negative pregnancy test complains of lower abdominal pain. What is the most appropriate work-up of this patient?
A. Complete blood count
B. Urinalysis
C. Abdominal and pelvic ultrasound
D. All of the above

9. A 33-year-old female with a history of ovarian cysts presents with an abrupt onset of severe right lower back, and right lower quadrant pain. Her urine hCG is negative. Her urine dipped positive for blood. Based on her clinical presentation and urinalysis, what is the most likely diagnosis?
A. Ureterolithiasis
B. Ruptured ovarian cyst
C. Hemorrhagic cyst
D. Appendicitis

10. A 27-year-old patient (G0P0) presents to emergency room because of left low quadrant pain for 2 weeks. She states that the pain is getting worse during the last 2 days. She has no fever, nausea, vomiting or diarrhea. The pain changes in the intensity. Pelvic examination reveals left adnexal mass, which is sensitive to palpation. Urine pregnancy test is negative. Pelvic ultrasound demonstrates a sonolucent cystic structure measuring $4.0 \times 4.7 \mathrm{~cm}$ with normal blood flow pattern. What is the most appropriate decision?
A. Perform immediate laparoscopy
B. Perform laparotomy
C. Repeat pelvic examination in 6 months
D. Repeat pelvic ultrasound in 6 weeks

\section{Answers}

$\begin{array}{llllllllllllllllllll}1 & \mathrm{D} & 2 & \mathrm{D} & 3 & \mathrm{C} & 4 & \mathrm{C} & 5 & \mathrm{C} & \mathbf{6} & \mathrm{B} & 7 & \mathrm{~A} & \mathbf{8} & \mathrm{D} & \mathbf{9} & \mathrm{A} & \mathbf{1 0} & \mathrm{D}\end{array}$

